\title{
Investigating analgesic and psychological factors associated with risk of postpartum depression development: a case-control study
}

This article was published in the following Dove Press journal:

Neuropsychiatric Disease and Treatment

9 June 2016

Number of times this article has been viewed

Thangavelautham

Suhitharan'

Thi Phuong Tu Pham ${ }^{2}$

Helen Chen ${ }^{2,3}$

Pryseley Nkouibert Assam ${ }^{4}$

Rehena Sultana ${ }^{2}$

Nian-Lin Reena $\mathrm{Han}^{5}$

Ene-Choo Tan ${ }^{6,7}$

Ban Leong Sng ${ }^{1,2}$

'Department of Women's Anaesthesia, KK Women's and Children's Hospital,

${ }^{2}$ Duke-NUS Medical School, ${ }^{3}$ Women's

Service, Department of Psychological

Medicine, KK Women's and Children's Hospital, ${ }^{4}$ Singapore Clinical Research Institute, ${ }^{5}$ Division of Clinical Support Services, ${ }^{6}$ Research Laboratory, KK Women's and Children's Hospital, ${ }^{7}$ SingHealth Paediatrics Academic Clinical Programme, Duke-NUS

Medical School, Singapore
Correspondence: Ban Leong Sng Department of Women's Anaesthesia, KK Women's and Children's Hospital, 100 Bukit Timah Road, Singapore 229899 , Singapore

$\mathrm{Tel}+656394$ l08।

$\mathrm{Fax}+65$ 629| 266।

Email sng.ban.leong@singhealth.com.sg
Aim: The aim of this study was to investigate the role of peripartum analgesic and psychological factors that may be related to postpartum depression (PPD).

Methods: This case-control study was conducted in pregnant females who delivered at KK Women's and Children's Hospital from November 2010 to October 2013 and had postpartum psychological assessment. Demographic, medical, and postpartum psychological status assessments, intrapartum data including method of induction of labor, mode of labor analgesia, duration of first and second stages of labor, mode of delivery, and pain intensity on hospital admission and after delivery were collected. PPD was assessed using the Edinburgh Postnatal Depression Scale and clinical assessment by the psychiatrist.

Results: There were 62 cases of PPD and 417 controls after childbirth within 4-8 weeks. The odds of PPD was significantly lower (33 of 329 [10.0\%]) in females who received epidural analgesia for labor compared with those who chose nonepidural analgesia (29 of 150 [19.3\%]) ([odds ratio] $0.47(0.27-0.8), P=0.0078)$. The multivariate analysis showed that absence of labor epidural analgesia, increasing age, family history of depression, history of depression, and previous history of PPD were independent risk factors for development of PPD.

Conclusion: The absence of labor epidural analgesia remained as an independent risk factor for development of PPD when adjusted for psychiatric predictors of PPD such as history of depression or PPD and family history of depression.

Keywords: epidural analgesia, childbirth, Edinburgh Postnatal Depression Scale

\section{Introduction}

Pregnancy and childbirth are significant events in a woman's life. Childbirth is associated with complex physiological, psychological, and social interaction. Extreme labor pain, complicated prolonged labor, and a sense of lack of control are known to traumatize the childbirth experience and lead to postpartum depression (PPD), which affects the health of mothers and their newborns and is associated with long-term psychological and socioeconomic impact. ${ }^{1,2}$

PPD is defined in the Diagnostic and Statistical Manual for Mental Disorders as major depression with postpartum onset with episodes of depression beginning within 4 weeks of giving birth. ${ }^{3}$ PPD is significant as it may last up to 6 months after delivery with significant public health impact on maternal health and child development. ${ }^{4}$ The incidence of PPD varies between studies depending on the time of survey postdelivery and population being investigated. PPD occurs in $\sim 10 \%-20 \%$ of females in the $\mathrm{US}^{5}$ and $\sim 8 \%$ of females in Singapore. ${ }^{6}$ A recent study assessing depressive symptoms using Edinburgh Postnatal Depression Scale (EPDS) in parturients at 
third trimester of pregnancy and 3 months postpartum by Verreault et $\mathrm{al}^{7}$ reported a higher rate of depressed mood up to $16.4 \%$ at 3 months postpartum. Another prospective cohort study in parturients admitted for a vaginal delivery by Ding et $\mathrm{al}^{8}$ reported the incidence of PPD was $24.3 \%$ at 6 weeks postpartum, when PPD was defined by a score of 10 or more on EPDS. Contributing factors for development of PPD are multifactorial, including psychological, physical, social, and obstetric factors. ${ }^{9,10}$ These factors include pain vulnerability and severity pain during childbirth, analgesic technique used during labor, and psychological and genetic susceptibility of the woman., ${ }^{9,11}$

Pain during childbirth has been described as one of the worst pain females may experience in her life. ${ }^{12}$ A longitudinal cohort study analyzing 1,288 deliveries in the US tertiary care center reported that one of five females after cesarean delivery and one of 13 females after vaginal delivery suffer from severe acute pain. ${ }^{13}$ The same study also identified severity of acute postpartum pain as an independent risk factor for development of persistent pain and depression. Previous studies have identified a strong link between the intensity of labor pain with posttraumatic stress and mood disorders in the early postpartum period. Soet et $\mathrm{al}^{14}$ described pain during labor was correlated with the development of posttraumatic stress disorder. Studies evaluating the severity of labor pain in the early postpartum period also found the correlation with postpartum blues ${ }^{15}$ and PPD. ${ }^{11}$ An earlier study by Hiltunen et $\mathrm{al}^{16}$ found that females who received epidural or paracervical block during their labor had lower depressive scores in the initial week after delivery compared with having no analgesia at all (odds ratio [OR] 0.25, 95\% confidence interval [CI] 0.09-0.72). However, paracervical block is rarely performed limiting its applicability to daily routine practice. On the other hand, these early results are consistent with the idea that more complete pain control (using epidural analgesia) may reduce PPD.

With modern obstetric practice, females have several pain relief options during childbirth. However, the implications of analgesic choice may have greater effect than pain relief itself. Epidural analgesia is considered to be the gold standard of pain relief during labor. This procedure effectively blocks the pain transmission through the spinal cord and yet allows mothers to participate fully in the laboring process with no sedation. In a recent prospective cohort study, Ding et $\mathrm{al}^{8}$ reported that epidural analgesia is associated with lower occurrence of PPD compared with those who did not receive any labor analgesia.

Parturients undergoing childbirth have several analgesic choices including epidural, opioid, and Entonox analgesia in current practice. As Ding et $\mathrm{al}^{8}$ compared epidural analgesia with no analgesia only, there is still a gap in knowledge with regard to other analgesic choices on the risk of PPD. Diagnosing PPD with EPDS, which is a screening tool, is also another controversial point that may overestimate the true incidence of PPD. Our study aims to investigate the role of potential peripartum analgesic and psychological factors that may be related to clinically confirmed PPD.

\section{Methods}

This study protocol was developed according to the Strengthening the Reporting of Observational studies in Epidemiology guidelines for reporting case-control studies and was reviewed and approved by the SingHealth Centralized Institutional Review Board. SingHealth Centralized Institutional Review Board registration: 2010/539/F. ClinicalTrials.gov registration: NCT02373709. Written informed consent was obtained from all the participants during the postpartum screening with EPDS.

This case-control study was conducted at KK Women's and Children's Hospital, Singapore from November 2010 to October 2013 in females who had their delivery and subsequently participated in the postpartum psychological assessment program. The exclusion criteria included adolescent mothers (age $<18$ years), females with stillbirth, or early neonatal loss. Medical records including epidural charts, postpartum monitoring charts, and the psychology notes at postpartum screening and consultation were reviewed. Information regarding peripartum events including peripartum obstetric data and PPD status was obtained for the data analysis.

We sought the following data from the available medical records: baseline data including age, weight, height, body mass index, and race. Socioeconomic status such as occupation, education level, type of housing, marital status, and number of children were self-reported. Obstetric history including parity, gestational age, and history of obstetric complications in current pregnancy including premature rupture of membrane were collected.

Intrapartum data including method of induction of labor, mode of labor analgesia, duration of first and second stages of labor, mode of delivery, and numeric rating scale pain score (11-point scale: 0 being no pain and 10 being the worst pain imaginable) on admission to labor ward and after delivery, occurrence of maternal pyrexia were documented.

The patients who came for routine postnatal consultations at the obstetrics clinics were screened and recruited via the postnatal screening program conducted by the Department of Psychological Medicine. Controls were obstetric patients who scored $<7$ on the EPDS. Cases were defined as patients 
with confirmed clinical diagnosis of PPD as assessed at the outpatient clinic by board-certified psychiatrists using the Diagnostic and Statistical Manual for Mental Disorders criteria for major depression of postpartum onset. ${ }^{17}$ These patients were referred from screening in the clinic or from the obstetric wards. Patients with psychotic disorders, comorbid predominant anxiety, or substance abuse were excluded. We used the Chinese version of EPDS validated in Singaporean peripartum females, if females could only speak Chinese. ${ }^{18}$

Data were summarized by cases (PPD) and controls (nonPPD) using mean (standard deviation) or median (range) for continuous variables as appropriate and number (proportions) of patients for categorical variables. OR together with corresponding 95\% CI were used to characterize the association between PPD development and demographic, maternal medical history, perinatal, analgesic, and psychological factors. Unadjusted and adjusted ORs were estimated using univariate and multivariate logistic regression analysis, respectively. The multivariate analysis was performed based on factors identified by univariate analyses with $P \leq 0.2$. Significance level was set at 5\%, and all tests were two-sided. SAS Version 9.3 software (SAS Institute Inc., Cary, NC, USA) was used for the analyses.

\section{Results}

Our data with a total of 744 patients who have delivered during the November 2010 to October 2013 and attended postpartum psychological assessment were reviewed in the study. Two hundred and fifty-four patients were excluded due to lack of labor analgesic data and eleven due to lack of confirmed PPD status. Data obtained from 479 patients were used for the analysis. There were 62 cases of PPD and 417 controls (without PPD) assessed within 4-8 weeks after childbirth.

The mean age (standard deviation) of the patients was 31.2 (4.9) years (range 19-48) (Table 1). Four hundred and seventy-two of 479 females $(98.5 \%)$ were Chinese. Almost all females were married; only one woman was single. Seventy-five percent of females had a university education, $23.8 \%$ had middle education such as secondary school or junior college, and $1.5 \%$ had basic education of primary school level. Sixty-three percent of these females

Table I Demographic characteristics $(n=479)$

\begin{tabular}{|c|c|c|c|c|}
\hline Characteristics & Case $(n=62)$ & Control $(n=4 \mid 7)$ & Unadjusted OR (95\% Cl) & P-value \\
\hline Age (years), mean (SD) & $33.76(6.25)$ & $30.84(4.6)$ & $2.92(1.62-4.21)$ & $<0.0001$ \\
\hline \multicolumn{5}{|l|}{ Chinese, n (\%) } \\
\hline No & $2(3.23)$ & $5(1.2)$ & Reference & \\
\hline Yes & $60(96.77)$ & $412(98.8)$ & $0.36(0.07-1.92)$ & 0.2260 \\
\hline Highest education level, n (\%) & & & & $0.0875^{c}$ \\
\hline Primary or below & $3(4.84)$ & $4(0.96)$ & $5.49(1.19-25.39)$ & 0.0385 \\
\hline Secondary/junior college & $16(25.81)$ & $98(23.5)$ & $1.20(0.65-2.22)$ & 0.1562 \\
\hline Postgraduate & $43(69.35)$ & $315(75.54)$ & Reference & \\
\hline Occupation, n (\%) & & & & 0.4572 \\
\hline Homemaker/unemployed & $23(37.1)$ & $123(29.5)$ & Reference & 0.2174 \\
\hline Management & $23(37.1)$ & $165(39.57)$ & $0.75(0.40-1.39)$ & 0.7541 \\
\hline Others & $16(25.81)$ & $129(30.94)$ & $0.66(0.34-1.32)$ & 0.3936 \\
\hline Marital status, n (\%) ${ }^{\mathrm{a}}$ & & & & $0.1294^{b}$ \\
\hline Married & 61 (98.39) & $417(100)$ & & \\
\hline Single & I (I.6I) & $0(0.0)$ & & \\
\hline Number of children, mean (SD) & $\mathrm{I} .73(0.89)$ & $1.48(0.72)$ & $1.46(1.07-2.00)$ & 0.0188 \\
\hline Weight (kg) - current, mean (SD) & $59.83(11.07)$ & $59.12(8.99)$ & $1.01(0.98-1.04)$ & 0.5762 \\
\hline Weight $(\mathrm{kg})$ - before pregnancy, mean (SD) & $55.83(10.55)$ & $54.22(9.58)$ & $1.02(0.99-1.04)$ & 0.2317 \\
\hline $\mathrm{BMI}$ - current, mean (SD) & $23.39(3.98)$ & $30.02(49.13)$ & $0.99(0.97-1.02)$ & 0.4581 \\
\hline BMI - before pregnancy, mean (SD) & $27.9(47.29)$ & $22.15(19.87)$ & $1.01(1.00-1.01)$ & 0.1536 \\
\hline \multicolumn{5}{|l|}{ Pregnancy, n (\%) } \\
\hline Planned-assisted & $36(58.06)$ & $266(63.79)$ & $0.79(0.46-1.35)$ & 0.3843 \\
\hline Unplanned & $26(41.94)$ & I5I (36.2I) & Reference & \\
\hline \multicolumn{5}{|l|}{ Expected childcare, n (\%) } \\
\hline Childcare/nursery & $30(48.39)$ & $258(61.87)$ & $0.58(0.34-0.99)$ & 0.0448 \\
\hline Self/partner & $32(51.6 I)$ & $159(38.13)$ & Reference & \\
\hline
\end{tabular}

Notes: Case represents patients with clinically confirmed diagnosis of PPD using DSM IV criteria of major depression of postpartum onset. Control represents non-PPD patients with EPDS score $\leq 7$. Unadjusted ORs were obtained from univariate logistic regression. ${ }^{a} O R$ cannot be estimated for marital status due to zero count. ${ }^{b} P$-value was calculated based on Fisher's exact test. 'Type III P-value.

Abbreviations: BMI, body mass index; Cl, confidence interval; DSM IV, Diagnostic and Statistical Manual for Mental Disorders, 4th edition; EPDS, Edinburgh Postnatal Depression Scale; OR, odds ratio; PPD, postpartum depression; SD, standard deviation. 
had their pregnancy planned, while 37\% did not plan their pregnancy. Sixty-two (12.9\%) patients were diagnosed with PPD. Univariate logistic regression analysis indicated that PPD was not significantly associated with females with high/ low economic status and high/basic education background, but was significantly associated with increasing age and number of children.

Table 2 shows 47 mothers (9.8\%) had medical complications during pregnancy. Among which, 33 females (6.9\%) had gestational diabetes mellitus and 14 females $(2.9 \%)$ had pregnancy-induced hypertension. However, there was no statistically significant difference between healthy mothers and mothers with medical complications in the outcome of PPD. Almost 10\% of mothers with PPD had a medical condition (diabetes mellitus, hypertension, and thyroid disease), whereas this number was much lower, $1.4 \%$, in the group without PPD. A univariate analysis showed that females who had a history of peripartum depression or depression in the past were more likely to develop PPD. Sixteen out of 26 patients $(61.5 \%)$ with a history of previous peripartum depression developed PPD, while only 46 out of 452 patients $(10.2 \%)$ without past history of peripartum depression developed PPD during this study period. Similarly, six out of nine patients $(66.7 \%)$ with past history of depression would develop PPD compared with 56 out of 469 (11.9\%) patients without past history of depression would develop PPD.

The mode of delivery, whether it was vaginal delivery, instrumental delivery, or emergency Cesarean section, was not associated with increased risk of PPD (Table 3). Perinatal events such as maternal pyrexia and premature rupture of membranes were not found to increase the risk of PPD. The duration of second stage of labor was significantly shorter in case group compared with control group. The numeric rating scale pain scores were similar between the PPD group and that of non-PPD group.

Table 4 shows epidural analgesia was performed in 329 females $(68.7 \%)$. The unadjusted odds of PPD was significantly lower in females who received epidural analgesia (33 of $329[10.0 \%]$ ) than those who did not (29 of 150 [19.3\%]) $(P=0.0078)$. The use of Entonox was not associated with decreased risk of PPD. The use of intramuscular pethidine on univariate analysis increased the risk of PPD. Nine of 32 females (28.1\%) who had intramuscular pethidine developed PPD, whereas 52 of 445 females (11.7\%) who did not receive intramuscular pethidine developed PPD.

Univariate logistic regression analysis showed that older age, multiple births, expected childcare by self/partner, any preexisting medical condition, any previous peripartum depression or any depression, any family history of depression, use of epidural and pethidine during epidural analgesia increased the risk of PPD. These risk factors and other potential confounders $(P \leq 0.2)$ were considered for the multivariate logistic regression. Table 5 shows multivariate logistic regression analysis identified five independent risk factors for PPD. The absence of epidural analgesia was associated with higher risk of depression (adjusted OR 1.95, 95\% CI 1.04-3.66, $P=0.0367)$. Increasing age was associated with higher risk of PPD (adjusted OR 1.56, 95\% CI 1.15-2.14, $P=0.005$, for every 5 years increase). Other psychological factors associated with higher risk of PPD were family

Table 2 Maternal medical history

\begin{tabular}{|c|c|c|c|c|}
\hline Characteristics & Case (n=62), n (\%) & Control ( $n=4 \mid 7), n(\%)$ & Unadjusted OR $(95 \% \mathrm{CI})$ & $P$-value* \\
\hline \multicolumn{5}{|c|}{ Pregnancy complication: gestational diabetes } \\
\hline No & $57(93.44)$ & $388(93.05)$ & Reference & \\
\hline Yes & $4(6.56)$ & $29(6.95)$ & $0.94(0.32-2.77)$ & 1.0000 \\
\hline \multicolumn{5}{|c|}{ Pregnancy complication: hypertension } \\
\hline No & $57(93.44)$ & $407(97.6)$ & Reference & \\
\hline Yes & $4(6.56)$ & $10(2.40)$ & $2.86(0.87-9.4 I)$ & 0.0898 \\
\hline \multicolumn{5}{|c|}{$\begin{array}{l}\text { Chronic medical conditions (diabetes mellitus, } \\
\text { hypertension, thyroid) }\end{array}$} \\
\hline No & $55(90.16)$ & $410(98.56)$ & Reference & \\
\hline Yes & $6(9.84)$ & $6(1.44)$ & $7.45(2.32-23.92)$ & 0.0017 \\
\hline \multicolumn{5}{|c|}{ Family history of depression } \\
\hline No & $48(77.42)$ & $406(97.6)$ & Reference & \\
\hline Yes & $14(22.58)$ & $10(2.40)$ & I I.84 (4.99-28.12) & $<0.000$ I \\
\hline \multicolumn{5}{|c|}{ History of depression } \\
\hline No & $56(90.32)$ & $413(99.28)$ & Reference & \\
\hline Yes & $6(9.68)$ & $3(0.72)$ & $14.75(3.59-60.64)$ & $<0.0001$ \\
\hline
\end{tabular}

Notes: Case represents patients with clinically confirmed diagnosis of PPD using DSM IV criteria of major depression of postpartum onset. Control represents non-PPD patients with EPDS score $\leq 7$. Unadjusted ORs were obtained from univariate logistic regression. *P-values were calculated based on Fisher's exact test.

Abbreviations: Cl, confidence interval; DSM IV, Diagnostic and Statistical Manual for Mental Disorders, 4th edition; EPDS, Edinburgh Postnatal Depression Scale; OR, odds ratio; PPD, postpartum depression. 
Table 3 Perinatal characteristics

\begin{tabular}{|c|c|c|c|c|}
\hline Characteristics & Case $(n=62)$ & Control $(n=4 \mid 7)$ & Unadjusted OR (95\% Cl) & $P$-value \\
\hline \multicolumn{5}{|c|}{ Vaginal delivery, n (\%) } \\
\hline No & $13(20.97)$ & $84(20.14)$ & Reference & \\
\hline Yes & $49(79.03)$ & $333(79.86)$ & $1.05(0.55-2.03)$ & $0.8663 *$ \\
\hline \multicolumn{5}{|c|}{ Instrumental delivery, $\mathrm{n}(\%)$} \\
\hline No & $42(87.50)$ & $28 I(84.13)$ & Reference & \\
\hline Yes & $6(12.50)$ & $53(15.87)$ & $0.76(0.3 \mathrm{I}-1.87)$ & $0.6717^{*}$ \\
\hline \multicolumn{5}{|c|}{ Cesarean section, n (\%) } \\
\hline No & $50(80.65)$ & $336(80.77)$ & Reference & \\
\hline Yes & $12(19.35)$ & $80(19.23)$ & $0.99(0.50-1.95)$ & $1.0000 *$ \\
\hline \multicolumn{5}{|c|}{ Maternal pyrexia, n (\%) } \\
\hline No & $59(95.16)$ & $382(91.61)$ & Reference & \\
\hline Yes & $3(4.84)$ & $35(8.39)$ & $\mathrm{I} .8(0.54-6.05)$ & $0.4533 *$ \\
\hline \multicolumn{5}{|c|}{ Premature rupture of membranes, $n(\%)$} \\
\hline No & $58(93.55)$ & $376(90.17)$ & Reference & \\
\hline Yes & $4(6.45)$ & $41(9.83)$ & $1.58(0.55-4.58)$ & $0.4904 *$ \\
\hline \multicolumn{5}{|c|}{ Duration of second stage labor (min) } \\
\hline Mean (SD) & $28.96(26.83)$ & $54.85(55.75)$ & $0.98(0.97-0.99)$ & $0.0025^{\#}$ \\
\hline \multicolumn{5}{|c|}{ Pain score recorded upon hospital admission } \\
\hline Mean (SD) & $3.60(3.91)$ & $4.4(3.81)$ & $0.95(0.88-1.02)$ & $0.1256^{\#}$ \\
\hline \multicolumn{5}{|c|}{ Pain score recorded during delivery } \\
\hline Mean (SD) & $0.10(0.65)$ & $0.29(\mathrm{I} .37)$ & $0.83(0.58-1.18)$ & $0.2977^{\#}$ \\
\hline $\begin{array}{l}\text { Notes: Case repres } \\
\text { patients with EPDS } \\
\text { calculated based on S }\end{array}$ & agnosis of PPD $u$ & IV criteria of major $\mathrm{d}$ & of postpartum onset. Control re & $\begin{array}{l}\text { s non-PPD } \\
\text { alues were }\end{array}$ \\
\hline Abbreviations: $\mathrm{Cl}$, & d Statistical Manu & tal Disorders, 4th editior & dinburgh Postnatal Depression Sca & odds ratio; \\
\hline
\end{tabular}

history of depression (adjusted OR 11.1, 95\% CI 4.23-29.75, $P<0.0001$ ), history of depression (adjusted OR 10.61, 95\% CI 2.34-58.15, $P=0.003$ ), and previous history of PPD (adjusted OR 9.42, 95\% CI 3.7-24.54, $P<0.0001$ ).

\section{Discussion}

Our study found that the odds of PPD were lower in females who received epidural analgesia for labor compared with those who chose nonepidural analgesia. We found a very strong association between development of PPD and previous history of depression or PPD and family history of depression.
Predisposing effect of these psychiatric risk factors has been well established and proved to be independent risk factors for PPD. ${ }^{10,19,20}$ Our finding of association between increasing maternal age and higher risk of PPD (OR 1.56 for every 5 -year increase) is similar to the finding of Canadian community health survey, which identified the prevalence of depression in females who had recently delivered was significantly higher in females aged 40-44 years than in females aged 30-35 years (OR 3.72, 95\% CI 2.15-6.41). ${ }^{21}$ This could be due to the fact that older females have higher rates of multiple births and pregnancy complications, such as high

Table 4 Primary outcome of PPD with regard to epidural analgesia and other modes of analgesia

\begin{tabular}{|c|c|c|c|c|c|}
\hline Mode of analgesia & Total $(n=479)$ & Case $(n=62)$ & Control $(n=417)$ & Unadjusted OR (95\% Cl) & $P$-value \\
\hline \multicolumn{6}{|l|}{ Epidural, n (\%) } \\
\hline With & $329(68.7)$ & $33(53.23)$ & $296(70.98)$ & $2.15(1.25-3.7)$ & 0.0078 \\
\hline Without & $150(31.3)$ & $29(46.77)$ & $121(29.02)$ & Reference & \\
\hline \multicolumn{6}{|l|}{ Entonox, n (\%) } \\
\hline With & $203(42.4)$ & $29(46.77)$ & $174(4 \mid .73)$ & $1.23(0.72-2.1)$ & 0.4921 \\
\hline Without & $276(57.6)$ & $33(53.23)$ & $243(58.27)$ & Reference & \\
\hline \multicolumn{6}{|l|}{ Pethidine, n (\%) } \\
\hline With & $32(8.5)$ & $9(14.52)$ & $23(5.53)$ & $2.90(1.28-6.6)$ & 0.0242 \\
\hline Without & $346(91.5)$ & $53(85.48)$ & $393(94.47)$ & Reference & \\
\hline
\end{tabular}

Notes: Case represents patients with clinically confirmed diagnosis of PPD using DSM IV criteria of major depression of postpartum onset. Control represents non-PPD patients with EPDS score $\leq 7$. Unadjusted ORs were obtained from univariate logistic regression. P-values were calculated based on Fisher's exact test.

Abbreviations: Cl, confidence interval; DSM IV, Diagnostic and Statistical Manual for Mental Disorders, 4th edition; EPDS, Edinburgh Postnatal Depression Scale; OR, odds ratio; PPD, postpartum depression. 
Table 5 Multivariate logistics regression for risk factors for PPD

\begin{tabular}{lll}
\hline Risk factors & $\begin{array}{l}\text { Adjusted } \\
\text { OR (95\% CI) }\end{array}$ & P-value \\
\hline Increase of maternal age* & I.56 (I.I5-2.I4) & 0.0050 \\
Epidural (no vs yes) & I.95 (I.04-3.66) & 0.0367 \\
$\begin{array}{l}\text { Family history of depression } \\
\text { (yes vs no) }\end{array}$ & I I.I (4.23-29.75) & $<0.000$ I \\
History of depression (yes vs no) & I0.6I (2.34-58.I5) & 0.003 I \\
$\begin{array}{l}\text { Previous peripartum depression } \\
\text { (yes vs no) }\end{array}$ & $9.42(3.7-24.54)$ & $<0.000$ I \\
\hline
\end{tabular}

Notes: Adjusted ORs were obtained from multivariate logistic regression by taking potential confounders $(P$-value $\leq 0.2)$ identified by univariate analysis into the calculation. *Every 5 year increase in age was associated with a higher risk of PPD.

Abbreviations: $\mathrm{Cl}$, confidence interval; OR, odds ratio; PPD, postpartum depression.

blood pressure and diabetes, which is associated with higher risk of PPD. We also proved the well-established independent psychiatric risk factors ${ }^{10,19}$ for the development of PPD, which includes family history of depression, history of depression, and previous history of PPD. The independent association between absence of labor epidural analgesia and development of PPD was confirmed using multivariate regression analysis including other risk factors such as maternal age and the above-mentioned psychological factors.

The main strength of the study is use of clinical assessment, which is the gold standard for the diagnosis of PPD. ${ }^{6}$ This would explain the lower overall rate of PPD in our study population (12.9\%) compared with the reported incidences of $24.3 \%$ from Asian study ${ }^{8}$ and $19.2 \%$ by a systematic review on incidence and prevalence of PPD. ${ }^{22}$ Most of these studies used EPDS to diagnose PPD. However, the researchers should note that the EPDS is not a diagnostic instrument, but rather a screening tool to detect depression in the postpartum period. Furthermore, a positive score could reflect a transient mood change rather than a depressive illness ${ }^{4}$ and eventually overestimate the incidence of PPD. A cutoff score of 12 or 13 is typically used for "probable depression", and as a marker for major depression, whereas a cutoff score of 9 or 10 is used for "possible" or minor depression. ${ }^{23}$

We found that the odds of PPD was lower in $10.0 \%$ (33/329) of females who received epidural analgesia compared with $19.3 \%$ (29/150) of those who choose nonepidural analgesia (OR $0.47(0.27-0.8), P=0.0078)$. Furthermore, absence of epidural analgesia was identified as a factor that could almost double the risk of developing of PPD. Our finding is consistent with the observational study by Ding et al, ${ }^{8}$ in which PPD occurred in 14.0\% (15 of 107) of females who received epidural labor analgesia and in 34.6\% (37 of 107) of those who did not have any analgesia $(P<0.001)$. They also found epidural analgesia was associated with a decreased risk of PPD compared with having no analgesia at all
(OR 0.31, 95\% CI 0.12-0.82, $P=0.018) .{ }^{8}$ However, our study methodology is different from Ding et $\mathrm{al}^{8}$ and Hiltunen et al. ${ }^{16}$ First, the diagnosis of PPD was made clinically by psychiatric specialist. Second, the above studies compared the effect of epidural analgesia on PPD with no analgesia at all. But we compared the epidural analgesia with nonepidural labor analgesic techniques such as Entonox or pethidine that replicates the real modern obstetric practice in many centers for labor analgesia, which is the strength of our study.

Limitations of our study are first due to the case-control design, where the exposure or outcome assessment maybe limited by recall bias of self-reported data during the psychiatric assessment and effect of unknown confounding factors. Most of the data during the labor were obtained from maternal monitoring chart, case note documents, and epidural charts. This would minimize the effect of recall bias. The clinical diagnosis of PPD by a specialist psychiatrist than EPDS will further reduce the effect of recall bias on the outcome. We had to exclude nearly one-third of the medical records reviewed to ensure the data accuracy. However, we managed to analyze 479 patients who provided adequate information and analysis for this case-control design. Furthermore, we cannot determine the causal relationship of epidural analgesia on development of PPD with this study design. The majority of our study population are Singaporean Chinese females (98.5\%) and no conclusions regarding females with different ethnic or socioeconomic groups may be drawn from this result. Lastly, we did not assess the psychological responses during labor that might be an important confounder. A woman who requested for epidural analgesia may have feelings of fear and insecurity, which might lead to increased risk of mood and depressive symptoms. The postpartum psychological assessment was done at 4-8 weeks with a median of 5 weeks postpartum and there is a possibility we could have missed detecting mild early PPD that resolved spontaneously during the psychological assessment.

Our study also showed other nonepidural labor analgesic techniques, like Entonox, mode of delivery, and pain scores after delivery, which were not associated with the development of PPD. The use of intramuscular pethidine may be associated with higher chance of development of PPD on the univariate analysis. This interesting finding corresponds to the published evidence from Robson and Kumar, ${ }^{24}$ who found poor maternal affection toward the new born and Mohammad et al, ${ }^{25}$ who described a significant association between development of PPD and pethidine use.

\section{Conclusion}

We conclude that use of labor epidural analgesia is associated with a reduction of the odds of PPD compared with 
nonepidural labor analgesic methods. Furthermore, absence of labor epidural analgesia is associated with the development of PPD. Prospective studies with larger sample size and a clinical confirmation of PPD is required to confirm the impact of epidural on PPD. These findings might help develop preventive strategies to decrease PPD through targeted evaluation and intervention. Further research is required to enable obstetricians and the anesthesiologists to have an evidence-based discussion with the pregnant females and the choice of epidural analgesia for labor pain.

\section{Acknowledgments}

We would like to acknowledge the administrative support from Ms Agnes Teo (Research Coordinator) and Ms Sarah Huan (Medical Student) and data collection by Jasmine $\mathrm{Ng}$ and Suzanne Tan (Research Assistants). We acknowledge the funding from the NMRC Clinical Trials Grant (CTG13feb013), NMRC Exploratory/Developmental Grant (NMRC/EDG/1006), and NMRC Centre Grant (NMRC/ $\mathrm{CG} / 006 / 2013$ ) in the conduct of research data collection and analysis for this manuscript.

\section{Author contributions}

All authors contributed toward data analysis, drafting, and revising the paper and agree to be accountable for all aspects of the work.

\section{Disclosure}

The authors report no conflicts of interest in this work.

\section{References}

1. Thompson KS, Fox JE. Post-partum depression: a comprehensive approach to evaluation and treatment. Ment Health Fam Med. 2010;7(4): 249-257.

2. Ballard CG, Stanley AK, Brockington IF. Post-traumatic stress disorder (PSTD) after childbirth. Br J Psychiatry. 1995;166:525-528.

3. Association AP. Diagnostic and Statistical Manual of Mental Disorders. 4th ed. Washington, DC: American Psychiatric Association; 1994.

4. Cox J, Holden J. Perinatal Mental Health: A Guide to the Edinburgh Postnatal Depression Scale. London: Gaskell Publishing; 2003.

5. Josefsson A, Berg G, Nordin C, Sydsjo G. Prevalence of depressive symptoms in late pregnancy and postpartum. Acta Obstet Gynecol Scand. 2001;80(3):251-255.
6. Chen H. Addressing maternal mental health needs in Singapore Psychiatr Serv. 2011;62(1):102.

7. Verreault N, Da Costa D, Marchand A, Ireland K, Dritsa M, Khalife S. Rates and risk factors associated with depressive symptoms during pregnancy and with postpartum onset. J Psychosom Obstet Gynaecol. 2014;35(3):84-91.

8. Ding T, Wang DX, Qu Y, Chen Q, Zhu SN. Epidural labor analgesia is associated with a decreased risk of postpartum depression: a prospective cohort study. Anesth Analg. 2014;119(2):383-392.

9. Halbreich U. Postpartum disorders: multiple interacting underlying mechanisms and risk factors. $J$ Affect Disord. 2005;88(1):1-7.

10. Robertson E, Grace S, Wallington T, Stewart DE. Antenatal risk factors for postpartum depression: a synthesis of recent literature. Gen Hosp Psychiatry. 2004;26(4):289-295.

11. Miller LJ. Postpartum depression. JAMA. 2002;287(6):762-765.

12. Melzack R. Labour pain as a model of acute pain. Pain. 1993;53(2): $117-120$.

13. Eisenach JC, Pan PH, Smiley R, Lavand'homme P, Landau R, Houle TT. Severity of acute pain after childbirth, but not type of delivery, predicts persistent pain and postpartum depression. Pain. 2008;140(1):87-94.

14. Soet JE, Brack GA, DiIorio C. Prevalence and predictors of women's experience of psychological trauma during childbirth. Birth. 2003; 30(1):36-46.

15. Boudou M, Teissedre F, Walburg V, Chabrol H. Association between the intensity of childbirth pain and the intensity of postpartum blues. Encephale. 2007;33(5):805-810.

16. Hiltunen P, Raudaskoski T, Ebeling H, Moilanen I. Does pain relief during delivery decrease the risk of postnatal depression? Acta Obstet Gynecol Scand. 2004;83(3):257-261.

17. Tan EC, Tan HS, Chua TE, et al. Association of premenstrual/menstrual symptoms with perinatal depression and a polymorphic repeat in the polyglutamine tract of the retinoic acid induced 1 gene. J Affect Disord. 2014;161:43-46.

18. Chee CY, Chong YS, Ng TP, Lee DT, Tan LK, Fones CS. The association between maternal depression and frequent non-routine visits to the infant's doctor - a cohort study. J Affect Disord. 2008;107(1-3): 247-253.

19. Beck CT. Predictors of postpartum depression: an update. Nurs Res. 2001;50(5):275-285.

20. Chaaya M, Campbell OM, El Kak F, Shaar D, Harb H, Kaddour A. Postpartum depression: prevalence and determinants in Lebanon. Arch Womens Ment Health. 2002;5:65-72.

21. Muraca GM, Joseph KS. The association between maternal age and depression. J Obstet Gynaecol Can. 2014;36(9):803-810.

22. Gavin NI, Gaynes BN, Lohr KN, Meltzer-Brody S, Gartlehner G, Swinson T. Perinatal depression: a systematic review of prevalence and incidence. Obstet Gynecol. 2005;106(5 Pt 1):1071-1083.

23. Gibson J, McKenzie-McHarg K, Shakespeare J, Price J, Gray R. A systematic review of studies validating the Edinburgh Postnatal Depression Scale in antepartum and postpartum women. Acta Psychiatr Scand. 2009;119(5):350-364.

24. Robson KM, Kumar R. Delayed onset of maternal affection after childbirth. Br J Psychiatry. 1980;136:347-353.

25. Mohammad KI, Gamble J, Creedy DK. Prevalence and factors associated with the development of antenatal and postnatal depression among Jordanian women. Midwifery. 2011;27(6):e238-e245.
Neuropsychiatric Disease and Treatment

\section{Publish your work in this journal}

Neuropsychiatric Disease and Treatment is an international, peerreviewed journal of clinical therapeutics and pharmacology focusing on concise rapid reporting of clinical or pre-clinical studies on a range of neuropsychiatric and neurological disorders. This journal is indexed on PubMed Central, the 'PsycINFO' database and CAS,

\section{Dovepress}

and is the official journal of The International Neuropsychiatric Association (INA). The manuscript management system is completely online and includes a very quick and fair peer-review system, which is all easy to use. Visit http://www.dovepress.com/testimonials.php to read real quotes from published authors. 